ARTICLE

https://doi.org/10.1038/s41467-020-18553-y

\title{
Electron hydrodynamics in anisotropic materials
}

\author{
Georgios Varnavides ${ }^{1,2,3,6}$, Adam S. Jermyn ${ }^{4,6}$, Polina Anikeeva (1) ${ }^{2,3}$, Claudia Felser $^{5}$ \& Prineha Narang (1) ${ }^{1 凶}$
}

Rotational invariance strongly constrains the viscosity tensor of classical fluids. When this symmetry is broken in anisotropic materials a wide array of novel phenomena become possible. We explore electron fluid behaviors arising from the most general viscosity tensors in two and three dimensions, constrained only thermodynamics and crystal symmetries. We find nontrivial behaviors in both two- and three-dimensional materials, including imprints of the crystal symmetry on the large-scale flow pattern. Breaking time-reversal symmetry introduces a non-dissipative Hall component to the viscosity tensor, and while this vanishes for 3D isotropic systems we show it need not for anisotropic materials. Further, for such systems we find that the electronic fluid stress can couple to the vorticity without breaking time-reversal symmetry. Our work demonstrates the anomalous landscape for electron hydrodynamics in systems beyond graphene, and presents experimental geometries to quantify the effects of electronic viscosity.

\footnotetext{
${ }^{1}$ Harvard John A. Paulson School of Engineering and Applied Sciences, Harvard University, Cambridge, MA 02138, USA. ${ }^{2}$ Department of Materials Science and Engineering, Massachusetts Institute of Technology, Cambridge, MA 02139, USA. ${ }^{3}$ Research Laboratory of Electronics, Massachusetts Institute of Technology, Cambridge, MA 02139, USA. ${ }^{4}$ Center for Computational Astrophysics, Flatiron Institute, New York, NY 10010, USA. ${ }^{5}$ Max-Planck-Institut für Chemische Physik fester Stoffe, Dresden 01187, Germany. ${ }^{6}$ These authors contributed equally: Georgios Varnavides, Adam S. Jermyn. ${ }^{凶}$ email: prineha@seas. harvard.edu
} 
T heoretical and experimental studies have revealed that electrons in condensed matter can behave hydrodynamically, exhibiting fluid phenomena such as Stokes flow and vortices ${ }^{1-9}$. Unlike classical fluids, preferred directions inside crystals lift isotropic restrictions, necessitating a generalized treatment of electron hydrodynamics. While anisotropic viscous flows have been studied in geophysics ${ }^{10}$, their prominence in condensed matter has yet to be explored. This is of particular importance, given the recent demonstration of hydrodynamic behavior in three-dimensional materials such as Weyl semimetals ${ }^{11,12}$. Electron hydrodynamics is observed when microscopic scattering processes conserve momentum over time- and length scales that are large compared to those of the experimental probe. However, even as momentum is conserved, free energy may be dissipated from the electronic system, giving rise to a measurable viscosity in the electron flow ${ }^{12-18}$.

When momentum is conserved, a fluid obeys Cauchy's laws of motion $^{19}$

$$
\begin{gathered}
\rho \dot{u}_{i}=\partial_{j} \tau_{j i}+\rho f_{i} \\
\rho \dot{\sigma}_{i}=\partial_{j} m_{j i}+\rho l_{i}+\epsilon_{i j k} \tau_{j k},
\end{gathered}
$$

where $u$ and $\rho$ are the fluid velocity and density, $f$ and $l$ are body forces and couples, $\tau$ and $m$ are the fluid stress and couple stress, and $\sigma$ is the intrinsic angular momentum density (internal spin). The superscript dot denotes the material derivative, $\dot{x}=\partial_{t} x+u_{j} \partial_{j} x$, and $\epsilon$ is the rank-3 alternating tensor. We assume couple stresses and body couples to be zero, but allow for body forces of the form $\rho f_{i}=-R_{i j} u_{j}$, where $R$ is a rank-2, positive-semidefinite tensor that is inversely proportional to a microscopic momentum-relaxing lifetime. In steady state and at experimentally accessible Reynolds numbers ${ }^{17,20}$, this implies that the stress tensor is symmetric ${ }^{19}$. In this limit, electron fluids obey the modified Navier-Stokes equation

$$
\rho u_{j} \partial_{j} u_{i}=-\partial_{i} p+\partial_{j} \tau_{j i}-R_{i j} u_{j},
$$

where $\tau$ is symmetric. Note that in electron fluids, current density is analogous to the fluid velocity, and voltage drops are analogous to changes in pressure. Assuming that the fluid velocity is much smaller than the electronic speed of sound, $u \ll c_{\mathrm{s}}$, the electron fluids are nearly incompressible, thus

$$
\partial_{i} u_{i}=0 .
$$

In this limit, $\rho$ is a constant, which we take to be unity. Since the fluid stress appears in a divergence, it is defined only up to a constant, which we choose to make $\tau$ vanish when $u$ is uniformly zero $^{21,22}$. We further assume that the fluid stress vanishes for uniform flow, so that it is only a function of the velocity gradient.

Without further loss of generality, the constitutive relation is written to the first order $a s^{21}$

$$
\tau_{i j}=A_{i j k l} \partial_{l} u_{k},
$$

where $A$ is the fluid viscosity, a rank-4 tensor relating the fluid velocity gradient $\left(\partial_{j} u_{i}\right)$ and the fluid stress. Since we take $\tau$ to be symmetric, $A$ is invariant under permutation of its first two indices, i.e., $A_{i j k l}=A_{i k k l} l^{21,22}$. Viscosity is represented as the sum of three rank-4 tensor basis elements ${ }^{23}$, summarized in Table 1

$$
A_{(i j) k l}=\alpha_{((i j)(k l))}+\beta_{[(i j)(k l)]}+\gamma_{(i j)[k l]} .
$$

Tensor $\alpha$ describes dissipative behavior respecting both stress symmetry and objectivity, i.e., $\alpha_{i j k l}=\alpha_{j i k l}=\alpha_{k l i j}$. Tensor $\beta$ on the other hand, describes nondissipative Hall viscosity ${ }^{7,23-27}$, i.e., $\beta_{i j k l}=-\beta_{k l i j}$, and is nonzero only when time-reversal symmetry is broken. Finally, $\gamma$ breaks stress objectivity, i.e., $\gamma_{i j k l}=-\gamma_{i j l k}$, coupling fluid stress to the vorticity. The fifth column in Table 1

\section{Table 1 Rank-4 tensors used as orthogonal basis elements for the viscosity tensor.}

\begin{tabular}{lllllll} 
Tensor & \multicolumn{3}{l}{ Tensor symmetries } & & \multicolumn{2}{l}{ Indep. comp. } \\
\hline & $i \leftrightarrow j$ & $k \leftrightarrow l$ & $i j \leftrightarrow k l$ & Type & $3 \mathrm{D}$ & $2 \mathrm{D}$ \\
$\alpha_{((i j)(k l))}$ & + & + & + & Proper & 21 & 6 \\
$\beta_{[(i j)(k l)]}$ & + & + & - & Pseudo & 15 & 3 \\
$\gamma_{(i j)[k l]}$ & + & - & $\mathrm{N} / \mathrm{A}$ & Pseudo & 18 & 3 \\
\hline
\end{tabular}

Even and odd symmetries are represented using parentheses and square brackets, respectively. The fifth column specifies whether the tensor changes sign under mirror operations.

specifies whether the tensor is defined according to a handedness convention.

In classical fluids, the added consideration of rotational invariance requires $A$ to be isotropic, reducing it to the form

$$
\begin{aligned}
A_{i j k l}= & \lambda \delta_{i j} \delta_{k l}+\mu\left(\delta_{i l} \delta_{j k}+\delta_{j l} \delta_{i k}\right) \\
& +\mathcal{B}_{1}\left(\epsilon_{i k} \delta_{j l}+\delta_{i k} \epsilon_{j l}\right)+\Gamma_{1} \delta_{i j} \epsilon_{k l},
\end{aligned}
$$

where $\delta$ is the Kronecker delta, $\epsilon$ is the rank-2 alternating tensor, and the Lamé parameters $\lambda$ and $\mu$ can be identified as the two independent components of the proper tensor $\alpha$. In the incompressible case, $\lambda$ does not contribute to the stress ${ }^{21} \cdot \mathcal{B}_{1}$ and $\Gamma_{1}$ are constants parameterizing terms with the symmetry of $\beta$ and $\gamma$, respectively. Since $\beta$ and $\gamma$ are pseudotensors, the last three terms in Eq. (7) are only nonzero in two dimensions ${ }^{23,24}$.

In crystals, however, there exist preferred directions and we cannot assume rotational invariance. Instead, we must consider the effect of the crystal symmetry given by Neumann's principle 28,29 , which requires that physical properties described by rank- 4 tensors, such as viscosity, remain invariant under the transformation law

$$
A_{i j k l}^{\prime}=|s|^{\eta} s_{i m} s_{j n} s_{k o} s_{l p} A_{m n o p},
$$

where $s$ is the space representation of any given point group symmetry of the crystal, $|s|= \pm 1$ is the determinant of the symmetry operation, and $\eta=0$ for proper tensors and $\eta=1$ for pseudotensors.

Although Eq. (8) relates different components of the viscosity tensor, further constrains must be imposed to ensure that the viscosity tensor never does positive work in Eq. (3), so that for any velocity field $u$ in $d$ dimensions

$$
\int u_{i} \partial_{j}\left(A_{i j k l} \partial_{k} u_{l}\right) d^{d} \mathbf{r} \leq 0
$$

Letting the Fourier transform of $u$ be

$$
\tilde{\mathbf{u}}(\mathbf{q})=\int e^{i \mathbf{q} \cdot \mathbf{r}} u(\mathbf{r}) d^{d} \mathbf{r}
$$

in $d$ dimensions, we find

$$
\int q_{j} q_{k} \tilde{u}_{i}^{*}(\mathbf{q}) \tilde{u}_{l}(\mathbf{q}) A_{i j k l} d^{d} \mathbf{q} \geq 0 .
$$

This is satisfied when $A_{i j k l}$ has a positive definite biquadratic form in $i l$ and $j k$, so we impose this constraint in addition to $i j$ symmetry and crystal symmetry.

Viscosity tensors are then randomly generated to satisfy the aforementioned constraints, allowing for normalized numerical deviations from isotropy lower than order unity. The viscosity tensor is assumed to be spatially uniform in all cases. To demonstrate the differences between these general viscosity tensors and those more strongly constrained by symmetry, we solve for the velocity and pressure of low Reynolds number flows in several geometries. The parameterization of the viscosity tensor in 
a

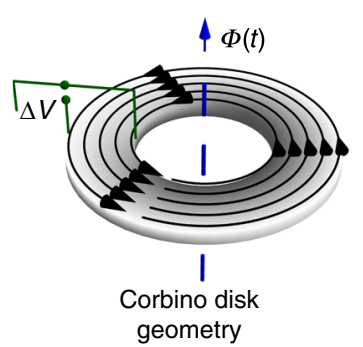

geometry

e

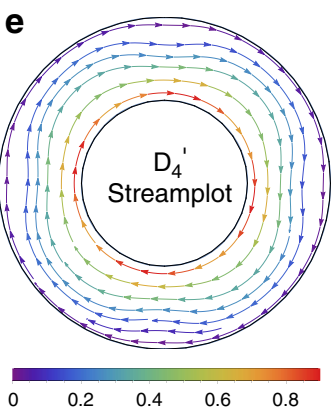

b

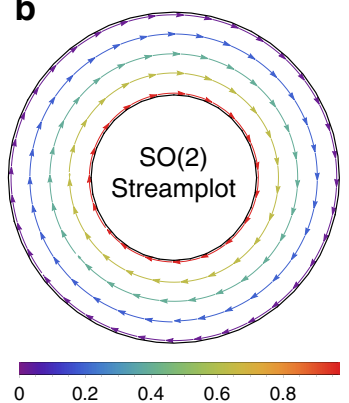

$\mathbf{f}$

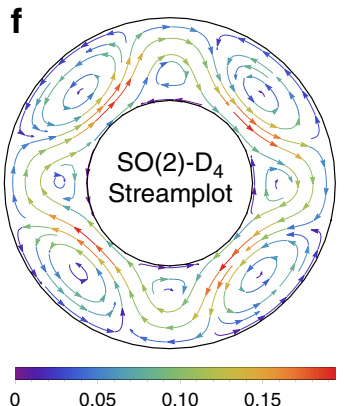

c

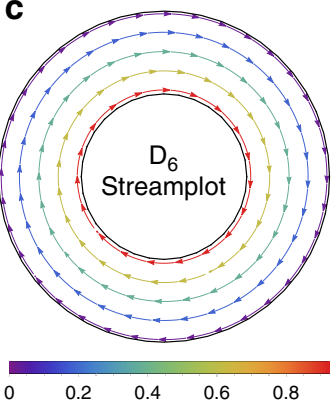

g

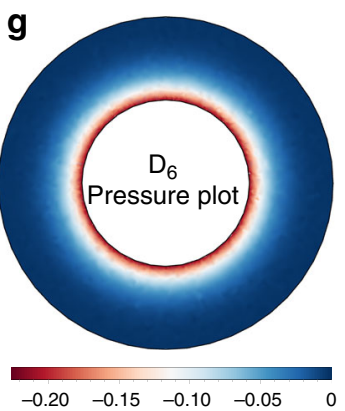

d

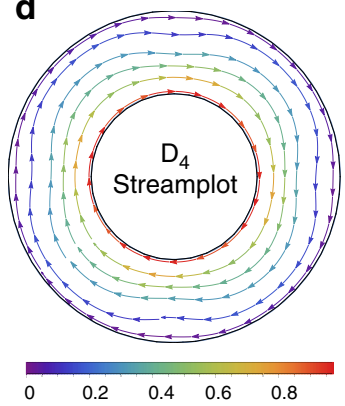

h

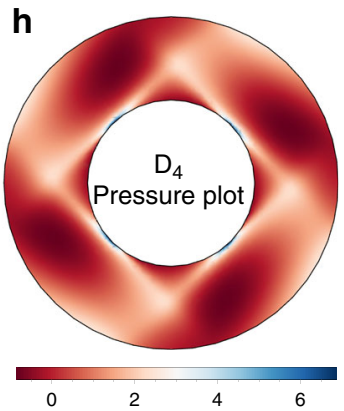

Fig. 1 Effect of viscosity tensor anisotropy on rotational flow in an annulus. a Corbino disk geometry schematic. The time-varying magnetic flux, $\Phi(t)$, acting on a voltage drop, $\Delta V$, gives rise to a Lorentz force, inducing rotational electron flow. $\mathbf{b}$ Steady-state streamplot plot using an isotropic (SO(2)) viscosity tensor. Streamplots using $\mathbf{c}$ hexagonal $\left(D_{6}\right)$, and $\mathbf{d}$ square $\left(D_{4}\right)$ viscosity tensors. e Streamplot using square $\left(D_{4}\right)$ viscosity tensor, allowing for momentum-relaxing body force terms equal to $|R| L^{2} /|A|=0.1$. f Difference in steady-state streamplot between isotropic and $D_{4}$ viscosity tensors,

highlighting the emergence of steady-state vortices. Steady-state pressure plot using $\mathbf{g} D_{6}$ and $\mathbf{h} D_{4}$ viscosity tensors, illustrating the breaking of azimuthal symmetry in the latter. Color scales indicate the magnitude of the velocity vector field (b-f) and pressure field $(\mathbf{g}, \mathbf{h})$.

Eq. (6) allows us to explore the effects of breaking stress objectivity and time-reversal symmetry. We highlight the effects of symmetry in the last two indices $(k l)$ because it implies that the stress only couples to the strain rate $\left(\partial_{k} u_{l}+\partial_{l} u_{k}\right)$ and not to the vorticity $\left(\partial_{k} u_{l}-\partial_{l} u_{k}\right)$. This is a property of classical fluids, which means that rigid-rotational flows are stress-free, and hence are only sensitive to rotation via weaker effects like the Coriolis force. Below, we demonstrate that with more general viscosity tensors, this is not the case, and that the resulting rotational stresses can be probed in experimentally accessible geometries.

\section{Results}

Effect of anisotropy. We first consider rotational flow in an annulus with inner radius $R_{\text {inner }}=1$ and outer radius $R_{\text {outer }}=2$ (Fig. 1). We apply a no-slip condition to the outer boundary, allow the inner boundary to rotate with unit angular velocity $\omega=$ 1, and solve for the steady-state flow at Reynolds number

$$
\operatorname{Re} \equiv \frac{\omega R_{\text {inner }}^{2}}{|A|}=0.3
$$

where

$$
|A|^{2}=A_{i j k l} A_{i j k l} \text {. }
$$

The zero-pressure point is fixed at the bottom of the annulus. Experimentally, such rotational flows can be achieved by threading a time-varying magnetic flux through a Corbino disk geometry 30,31 (Fig. 1a). For a fluid with an isotropic viscosity, the steady-state velocity field rotates rigidly with the angular velocity set by the inner boundary condition (Fig. 1b).

To investigate the effects of anisotropy in two-dimensional materials, we consider materials with $D_{6}$ (hexagonal) and $D_{4}$ (square) symmetry. Notably, $D_{6}$ materials do not deviate from isotropic behavior (Fig. 1(c)), consistent with experimental observations for graphene ${ }^{9,17}$. We note that $2 \mathrm{D}$ materials with
$C_{3}$ (threefold), $C_{6}$ (sixfold), and $D_{3}$ (triangular) symmetry also exhibit isotropic viscosity tensors (see Supplementary Methods). By contrast, the flow deviates considerably from isotropic behavior in $D_{4}$ materials (Fig. 1d). We repeat the calculation, allowing for a momentum-relaxing body force equal to $|R| L^{2} /|A|$ $=0.1$, illustrating that the deviation from isotropy remains observable (Fig. 1e). We assume $\boldsymbol{R} \rightarrow 0$ for the rest of the paper, and investigate its effects and symmetry in Supplemenentary Figs. 1 and 2. Figure if shows the steady-state velocity flow difference between the isotropic case and $D_{4}$ materials. We observe steady-state vortices emerging at $\sim 15 \%$ of the bulk flow rate overlaid onto the isotropic velocity field. While the steadystate pressure field in $D_{6}$ materials mirrors that of an isotropic fluid (Fig. 1g), the pressure field in $D_{4}$ materials also exhibits four vortices (Fig. 1h), with orientation set by the underlying crystal axes.

Effect of asymmetry. We next examine the importance of symmetry in the last two indices of the viscosity tensor. We calculate the flow profile for the annulus in Fig. 1 scaled by a factor of two, equipped with a pressure gauge, as shown in Fig. 2a. The pressure gauge is a channel with no-slip boundary conditions, allowing us to measure the difference between the flow and a nearly stationary fluid. To isolate the effects of $\mathcal{B}_{1}$ and $\Gamma_{1}$ in Eq. (7), Fig. 2a, $\mathrm{b}$ shows the flow and pressure fields in the annulus for a material with isotropic viscosity tensor where both $\mathcal{B}_{1}$ and $\Gamma_{1}$ have been set to zero $(\mathrm{SO}(2)\{\alpha\})$. These are nearly unchanged inside the annulus as compared to Fig. 1b, g, with a constant pressure in the gauge. Allowing for nonzero stress-breaking components, i.e., using a material with isotropic viscosity for $\mathcal{B}_{1}=0$ and $\Gamma_{1}=0.25$ $(\mathrm{SO}(2)\{\gamma\})$, we observe a significant pressure buildup near the gauge. This is due to the shear stress between the rotating and stationary fluids, while the pressure within the gauge itself is nearly uniform, as shown in Fig. 2c. 
a

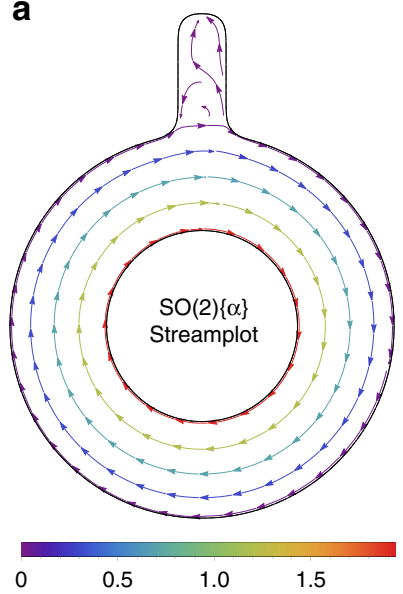

b

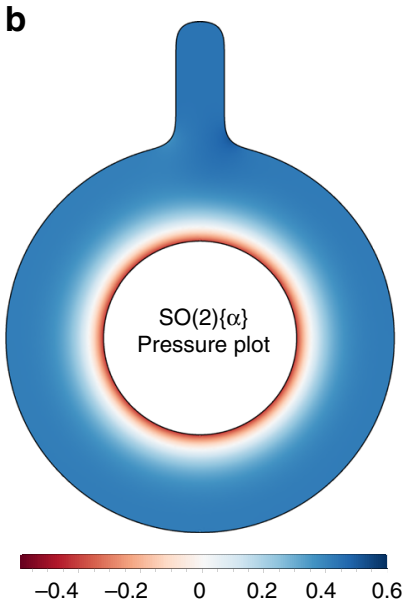

C

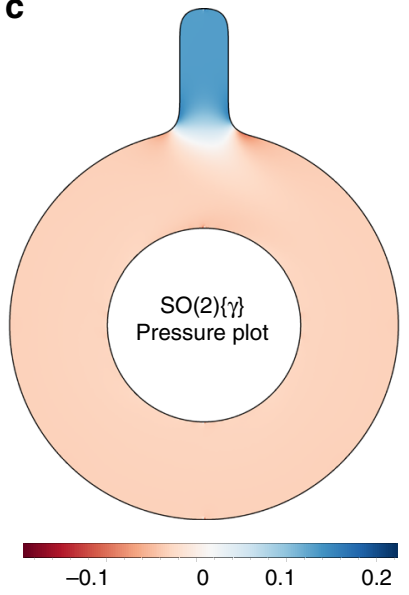

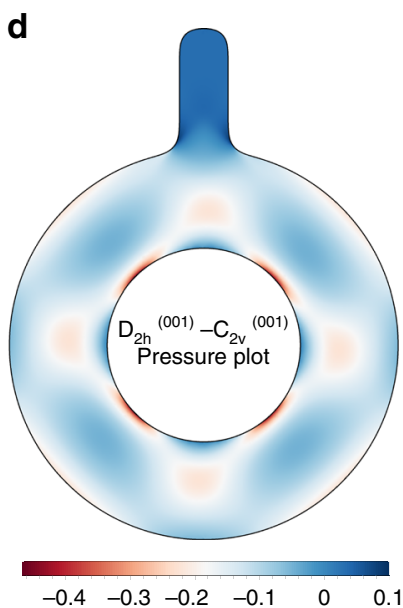

Fig. 2 Proposed setup to quantify the effect of viscosity tensor asymmetry and Hall coefficient. Steady-state $\mathbf{a}$ streamplot and $\mathbf{b}$ pressure plot using viscosity tensor $\mathrm{SO}(2)\{\alpha\}$. c Difference in steady-state pressure between viscosity tensor $\mathrm{SO}(2)\{\alpha\}$ and the same with additional stress objectivitybreaking terms $(\mathrm{SO}(2)\{\gamma\})$. The asymmetry introduces an additional pressure-like contribution, which can be directly measured. $\mathbf{d}$ Difference in steadystate pressure between the $D_{2 h}$ and $C_{2 v}$ viscosity tensors along the $a b$ plane, showing a similar pressure drop in the gauge, despite the anisotropic behavior inside the annulus. Color scales indicate the magnitude of the velocity vector field (a) and pressure field (b-d).

To quantify the pressure difference between $\mathrm{SO}(2)\{\alpha\}$ and $\mathrm{SO}$ (2) $\{\gamma\}$, note that the pressure is fixed to zero at a point $p$, the bottom of the annulus domain. The pressure in the gauge may be written as the path integral

$$
p_{\text {gauge }}=\int_{p}^{g} \nabla p \cdot d \mathbf{s}
$$

where $g$ is a point in the gauge. At low Reynolds numbers, we may neglect $u_{j} \partial_{j} u_{i}$ in Eq. (3), to find in the steady state

$$
\begin{gathered}
\nabla p=A_{i j k l} \partial_{i} \partial_{k} u_{l} . \\
p_{\text {gauge }}=\int_{p}^{g} A_{i j k l} \partial_{i} \partial_{k} u_{l} d s_{j} .
\end{gathered}
$$

Taking into account Eq. (7) and noting that the changes in fluid flow are negligible, we find

$$
\Delta p_{\text {gauge }}=\int_{p}^{g} \Delta A_{i j k l} \partial_{i} \partial_{k} u_{l} d s_{j}=\Gamma_{1} \int_{p}^{g} \partial_{i} \omega d s_{i}=\Gamma_{1} \Delta \omega
$$

where $\omega_{i}=\epsilon_{i j k} \partial_{j} u_{k}$ is the vorticity of the flow. For the geometry used, we find $\Delta p_{\text {gauge }}=0.15$, vorticity in the gauge is zero, and that in the annulus is 0.6 , so $\Delta \omega=0.6$ (Fig. 2c). Since we chose $\Gamma_{1}=0.25$, we see that in this setup, the pressure gauge $\left(\Delta p_{\text {gauge }}=\right.$ $\left.\Gamma_{1} \Delta \omega=0.25 \times 0.6=0.15\right)$ is directly sensitive to the asymmetry in the viscosity, which couples the rotation directly to the pressure field and the stress. We note that the same setup is sensitive to Hall viscosity coefficients for time-reversal broken systems, i.e., for the case where both $\mathcal{B}_{1}$ and $\Gamma_{1}$ are nonzero, the pressure gauge generalizes to

$$
\Delta p_{\text {gauge }}=\int_{p}^{g} \Delta A_{i j k l} \partial_{i} \partial_{k} u_{l} d s_{j}=\left(\mathcal{B}_{1}+\Gamma_{1}\right) \Delta \omega
$$

While time-reversal and stress objectivity-breaking terms persist in two-dimensional isotropic materials, the handedness of the pseudotensor implies that mirror operations set them to zero in 3D. This can be directly observed by comparing low- and high-symmetry three-dimensional crystals. We consider the same rotational flow along the $a b$ crystal plane of orthorhombic materials, such as the hydrodynamically reported Weyl semimetal WP2 ${ }^{11,12}$. Along this plane, the difference between the two viscosity tensors can be parameterized as follows:

$$
\begin{gathered}
A_{i j k l}^{C_{2 v l}^{(001)}}=A_{i j k l}^{D_{2 h}^{(001)}}+\Gamma_{2} \delta_{i j} \epsilon_{k l}+\Gamma_{3} \sigma_{i j}^{z} \epsilon_{k l} \\
1 e m+\mathcal{B}_{2}\left(\delta_{l i} \epsilon_{j k}-\epsilon_{l i} \delta_{j k}\right)+\mathcal{B}_{3}\left(\delta_{i j} \sigma_{k l}^{x}-\sigma_{i j}^{x} \delta_{k l}\right),
\end{gathered}
$$

where $\mathcal{B}_{2}, \mathcal{B}_{3}, \Gamma_{2}$, and $\Gamma_{3}$ are constants parameterizing terms with the symmetry of $\beta$ and $\gamma$, respectively, $\sigma^{x}$ and $\sigma^{z}$ are Pauli matrices. Figure $2 \mathrm{~d}$ shows the pressure difference between a material with $D_{2 h}$ symmetry and one with $C_{2 v}$ symmetry (for $\mathcal{B}_{2}=\mathcal{B}_{3}=\Gamma_{3}=0$ and $\Gamma_{2}=0.25$ ), indicating the same pressure buildup as in Fig. $2 \mathrm{c}$ inside the gauge along with a nontrivial pressure structure in the annulus.

2D flows in 3D crystals. Finally, we consider flow through an expanding channel along high-symmetry planes in 3D. This geometry has been proposed as a diagnostic of electron hydrodynamics because it naturally generates vortices, not present in ordinary ohmic flow. The case with isotropic viscosity is shown in Fig. 3a, where the small vortices that form in the corners are clearly detached from the bulk of the flow. We consider the $T_{\mathrm{d}}$ (tetrahedral) and $\mathrm{O}_{\mathrm{h}}$ (cubic) point groups. In particular, we consider flows along the polar $\{111\}$, nonpolar $\{110\}$, and semipolar $\{001\}$ family of planes (Fig. 3b)

$$
\begin{aligned}
& A_{i j k l}^{T_{\mathrm{d}}^{(111)}}=A_{i j k l}^{O_{1}^{(111)}}+\mathcal{B}_{4}\left(\sigma_{i j}^{x} \sigma_{k l}^{z}-\sigma_{i j}^{z} \sigma_{k l}^{x}\right)+\Gamma_{4} \delta_{i j} \epsilon_{k l} \\
& A_{i j k l}^{T_{\mathrm{d}}^{(110)}}=A_{i j k l}^{O_{\mathrm{h}}^{(110)}} \\
& A_{i j k l}^{T_{\mathrm{d}}^{(001)}}=A_{i j k l}^{O_{\mathrm{h}}^{(001)}}+\mathcal{B}_{5}\left(\sigma_{i j}^{z} \delta_{k l}^{z}-\delta_{i j} \sigma_{k l}^{z}\right)+\Gamma_{5} \sigma_{i j}^{x} \epsilon_{k l} .
\end{aligned}
$$

Along these planes, the difference between the two viscosity tensors can be parameterized according to Eqs. (20a), (20b), (20c). We impose fully developed (parabolic) inlet and outlet flows with constant discharge, and solve for the steady-state flow at low Reynolds number. Figure $3 \mathrm{c}$ shows the difference between the flow in an isotropic material and the flow in a cubic material along a $\{111\}$ close-packed plane, which exhibits rotational invariance. Along the nonpolar $\{110\}$ planes, terms with $\beta$ and $\gamma$ symmetry vanish. However, $A_{\mathrm{h}}^{O_{\mathrm{h}}^{(110)}}$ is anisotropic along this plane, with Fig. 3d showing the difference in flow between the isotropic 
a

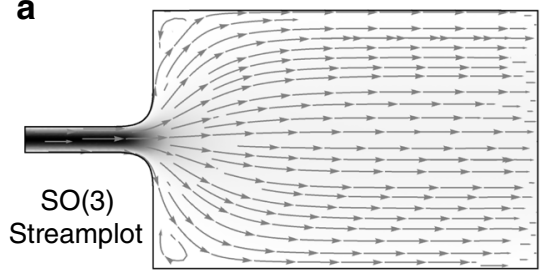

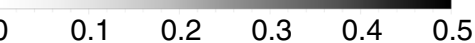

d

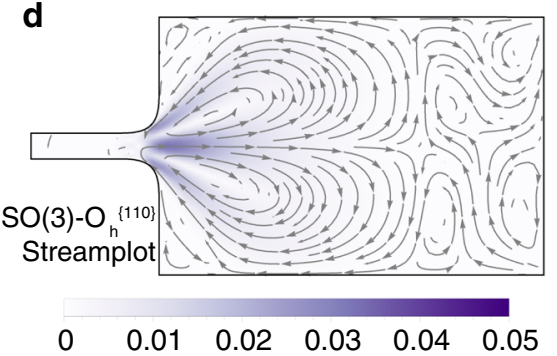

b

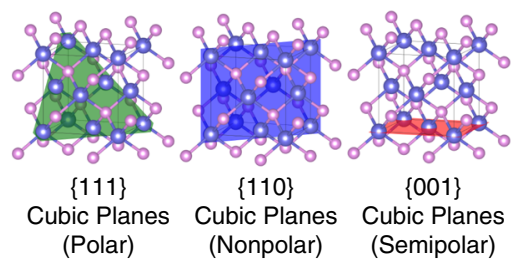

$\mathbf{e}$

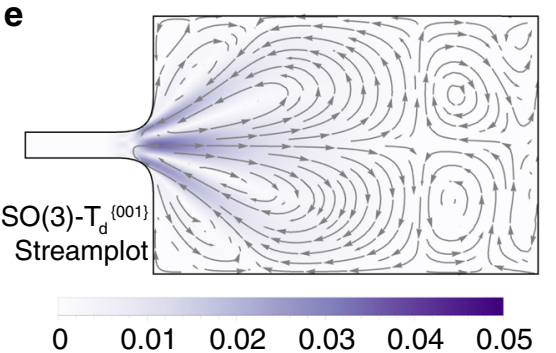

C

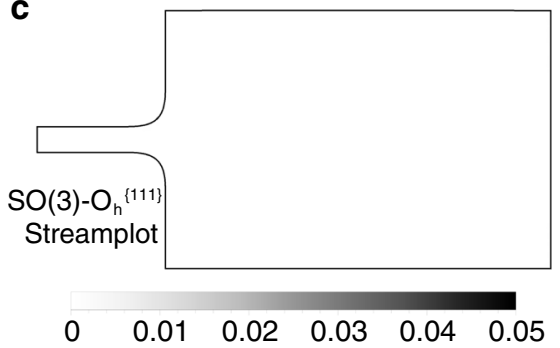

f

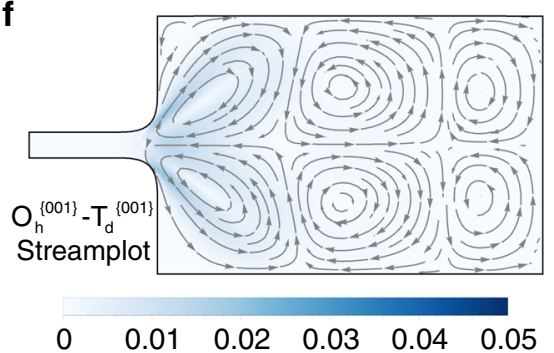

Fig. 3 Three-dimensional projected flows through an expanding geometry. a Steady-state streamplot using an isotropic (SO(3)) viscosity tensor. $\mathbf{b}$ Highsymmetry family of planes in cubic crystals. In crystals with $T_{\mathrm{d}}$ (tetrahedral) symmetry, these are further identified as polar $\{111\}$, nonpolar $\{110\}$, and semipolar \{001\}. Difference in the steady-state streamplot between using an isotropic viscosity tensor and using $\mathbf{c}$ (111)-projected, $\mathbf{d}$ (110)-projected, and $\mathbf{e}$ (001)-projected viscosity tensors of cubic crystals with $T_{d}$ symmetry. Note that $\mathbf{c}$ shows no difference. $\mathbf{f}$ Effect of viscosity tensor asymmetry difference between $O_{\mathrm{h}}$ and $T_{\mathrm{d}}$ crystals along a semipolar $\{001\}$ plane. Color scales indicate the magnitude of the velocity vector field.

case. Finally, along the semipolar $\{001\}$ family of planes, the viscosity tensor is both anisotropic (Fig. 3e) and asymmetric. Figure $3 \mathrm{f}$ quantifies the additional vortices generated by the asymmetry at $\sim 10 \%$, for $\mathcal{B}_{5}=0$ and $\Gamma_{5}=0.25$.

\section{Discussion}

We found that electron fluids in crystals with anisotropic and asymmetric viscosity tensors can exhibit steady-state fluid behaviors not observed in classical fluids. In $3 \mathrm{D}$, discrete deviations from isotropy allow the fluid stress to couple to the fluid vorticity with or without breaking time-reversal symmetry, for the case of Hall viscosity and objectivity-breaking viscosity, respectively. Recent measurements of spatially resolved flows $9,17,32$ suggest that these effects can be directly observed in systems beyond graphene. Our findings further hint at potential applications. For instance, the pressure gauge in Fig. 2 could be used as a magnetometer, converting a time-varying magnetic flux through a modified Corbino disk geometry into current in the annulus, and ultimately into a voltage drop between it and the gauge. Our work highlights the importance of crystal symmetry on electronic flow, and invites further exploration of time-dependent flows in systems with internal spin degrees of freedom and asymmetric stress tensors.

\section{Data availability}

The authors declare that the main data supporting the findings of this study are available within the article and its Supplementary Information files.

\section{Code availability}

Code available upon request from the authors.

Received: 24 May 2020; Accepted: 25 August 2020;

Published online: 18 September 2020

\section{References}

1. Andreev, A. V., Kivelson, S. A. \& Spivak, B. Hydrodynamic description of transport in strongly correlated electron systems. Phys. Rev. Lett. 106, 256804 (2011).
2. Levitov, L. \& Falkovich, G. Electron viscosity, current vortices and negative nonlocal resistance in graphene. Nat. Phys. 12, 672-676 (2016).

3. Scaffidi, T., Nandi, N., Schmidt, B., Mackenzie, A. P. \& Moore, J. E. Hydrodynamic electron flow and hall viscosity. Phys. Rev. Lett. 118, 226601 (2017).

4. Lucas, A. \& Fong, K. C. Hydrodynamics of electrons in graphene. J. Phys. 30, 053001 (2018).

5. Link, J. M., Narozhny, B. N., Kiselev, E. I. \& Schmalian, J. Out-of-bounds hydrodynamics in anisotropic dirac fluids. Phys. Rev. Lett. 120, 196801 (2018).

6. Cook, C. Q. \& Lucas, A. Electron hydrodynamics with a polygonal fermi surface. Phys. Rev. B 99, 235148 (2019).

7. Holder, T., Queiroz, R. \& Stern, A. Unified description of the classical hall viscosity. Phys. Rev. Lett. 123, 106801 (2019).

8. Holder, T. et al. Ballistic and hydrodynamic magnetotransport in narrow channels. Phys. Rev. B 100, 245305 (2019).

9. Sulpizio, J. A. et al. Visualizing poiseuille flow of hydrodynamic electrons. Nature 576, 75-79 (2019)

10. Perry-Houts, J. \& Karlstrom, L. Anisotropic viscosity and time-evolving lithospheric instabilities due to aligned igneous intrusions. Geophys. J. Int. 216, 794-802 (2018).

11. Coulter, J., Sundararaman, R. \& Narang, P. Microscopic origins of hydrodynamic transport in the type-II weyl semimetal wp. Phys. Rev. B 98, 115130 (2018).

12. Gooth, J. et al. Thermal and electrical signatures of a hydrodynamic electron fluid in tungsten diphosphide. Nat. Commun. 9, 4093 (2018).

13. Molenkamp, L. W. \& de Jong, M. J. M. Observation of knudsen and gurzhi transport regimes in a two-dimensional wire. Solid-State Electron. 37, 551-553 (1994).

14. de Jong, M. J. M. \& Molenkamp, L. W. Hydrodynamic electron flow in highmobility wires. Phys. Rev. B 51, 13389-13402 (1995).

15. Bandurin, D. A. et al. Negative local resistance caused by viscous electron backflow in graphene. Science 351, 1055-1058 (2016).

16. Crossno, J. et al. Observation of the dirac fluid and the breakdown of the wiedemann-franz law in graphene. Science 351, 1058-1061 (2016).

17. Ku, M. J. H. et al. Imaging Viscous Flow of the Dirac Fluid in Graphene Using a Quantum Spin Magnetometer. http://arxiv.org/abs/1905.10791v1.

18. Moll, P. J. W., Kushwaha, P., Nandi, N., Schmidt, B. \& Mackenzie, A. P. Evidence for hydrodynamic electron flow in PdCoO2. Science 351, 1061-1064 (2016).

19. Stokes, V. K. Couple stresses in fluids. Phys. Fluids 9, 1709 (1966).

20. Mendoza, M., Herrmann, H. J. \& Succi, S. Hydrodynamic model for conductivity in graphene. Sci. Rep. 3, 1052 (2013).

21. Landau, L. D. \& Lifshitz, E. M. Fluid Mechanics (London, Pergamon Press, 1959).

22. Steinberg, M. S. Viscosity of the electron gas in metals. Phys. Rev. 109, 1486-1492 (1958). 
23. Epstein, J. M. \& Mandadapu, K. K. Time Reversal Symmetry Breaking in Twodimensional Non-equilibrium Viscous Fluids. http://arxiv.org/abs/ 1907.10041v1.

24. Avron, J. E. Odd Viscosity. J. Stat. Phys. 92, 543-557 (1998).

25. Banerjee, D., Souslov, A., Abanov, A. G. \& Vitelli, V. Odd viscosity in chiral active fluids. Nat. Commun. 8, 1573 (2017).

26. Berdyugin, A. I. et al. Measuring Hall viscosity of graphene's electron fluid. Science 364, 162-165 (2019).

27. Rao, P. \& Bradlyn, B. Hall viscosity in quantum systems with discrete symmetry: point group and lattice anisotropy. Phys. Rev. X 10, 021005 (2020).

28. Neumann, F. E. Vorlesungen üBer die Theorie der Elastizität der Festen Körper und des Lichtäthers. (B. G. Teubner-Verlag, 1885).

29. Nye, J. F. Physical Properties of Crystals (Oxford University Press, 1985).

30. Tomadin, A., Vignale, G. \& Polini, M. Corbino disk viscometer for $2 \mathrm{~d}$ quantum electron liquids. Phys. Rev. Lett. 113, 235901 (2014).

31. Mumford, S., Paul, T., Lee, S. H., Yacoby, A. \& Kapitulnik, A. A cantilever torque magnetometry method for the measurement of hall conductivity of highly resistive samples. Rev. Sci. Instrum. 91, 045001 (2020).

32. Ella, L. et al. Simultaneous voltage and current density imaging of flowing electrons in two dimensions. Nat. Nanotechnol. 14, 480-487 (2019).

\section{Acknowledgements}

The authors thank Prof. Andrew Lucas of the University of Colorado Boulder for fruitful discussions. The authors acknowledge funding from the Defense Advanced Research Projects Agency (DARPA), Defense Sciences Office (DSO) Driven, and Nonequilibrium Quantum Systems program Grant Number D18AC00014 and the STC Center for Integrated Quantum Materials, NSF Grant No. DMR-1231319. A.S.J. is supported by the Flatiron Institute of the Simons Foundation. P.N. is a Moore Inventor Fellow supported by the Gordon and Betty Moore Foundation Grant Number 8048 .

\section{Author contributions}

G.V. and A.S.J. jointly conceived the ideas and developed the framework. G.V., C.F., and P.N. identified the material systems and link with the transport measurements of topological systems. G.V., P.A., and P.N. jointly worked on introducing the effects of crystal symmetries. All authors discussed the findings and contributed to the writing of the paper.

\section{Competing interests}

The authors declare no competing interests.

\section{Additional information}

Supplementary information is available for this paper at https://doi.org/10.1038/s41467020-18553-y.

Correspondence and requests for materials should be addressed to P.N.

Peer review information Nature Communications thanks Gregory Falkovich and the other, anonymous, reviewer(s) for their contribution to the peer review of this work. Peer reviewer reports are available.

Reprints and permission information is available at http://www.nature.com/reprints

Publisher's note Springer Nature remains neutral with regard to jurisdictional claims in published maps and institutional affiliations.

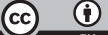

Open Access This article is licensed under a Creative Commons Attribution 4.0 International License, which permits use, sharing, adaptation, distribution and reproduction in any medium or format, as long as you give appropriate credit to the original author(s) and the source, provide a link to the Creative Commons license, and indicate if changes were made. The images or other third party material in this article are included in the article's Creative Commons license, unless indicated otherwise in a credit line to the material. If material is not included in the article's Creative Commons license and your intended use is not permitted by statutory regulation or exceeds the permitted use, you will need to obtain permission directly from the copyright holder. To view a copy of this license, visit http://creativecommons.org/ licenses/by/4.0\%

(C) The Author(s) 2020 